\title{
Development of 3D Virtual Models and 3D Construction Methods for Garments
}

\author{
Ei C. HLAING*a, Sybille KRZYWINSKI ${ }^{a}$, Hartmut ROEDEL ${ }^{a}$ \\ ${ }^{a}$ Institute of Textile Machinery and High Performance Material Technology \\ Technical University of Dresden, Germany
}

\begin{abstract}
The requirements for the clothing industry have altered over the years. The production has to be changed from the standard mass production to the production of fast changing trendy range of products. Causations for this are market saturation, import pressure and constantly changing consumer preferences. In addition to a variety of fashionable materials and styles, high functionality and perfect fit of clothing are requisite. Due to the enormous competitive pressure it is imperative to reduce time and costs of product development in the clothing industry.

The aim of the research project is to develop 3D virtual models of lower bodies from the scanned data of different body types as a prerequisite for computer-aided 3D product development of loose-fitting garments.

As an example, lady trousers are constructed. In favour of the reproducible construction of fashionable/functional outerwear on the basis of automatically generated models, 3D construction algorithms based on commercially available software solutions have to be compiled and development tools have to be created. In doing so geometrical as well as functional variable parameters are predefined. The trouser can be modified by changing predefined parameters and can then be used as a basis for constructing the variety of trousers. Two-dimensional (2D) pattern pieces are automatically generated and modified if necessary. According to morphological changes the whole process proceeds automatically up to $2 \mathrm{D}$ patterns and thus corresponds to a grading in $3 \mathrm{D}$.
\end{abstract}

Keywords: 3D virtual models, computer-aided 3D product development, loose-fitting garment, 2D pattern, grading in $3 \mathrm{D}$

\section{State of the art}

In constructing the loose-fitting garment based on the currently used 2D Pattern Making Systems (2DCAD), the skill and experience of the designer plays an important role to achieve the desired product of well fit and high quality product. The task of the designer is to transfer three-dimensional body shape into two-dimensional pattern pieces taking into account physical and fit related design features. Since the link between the three-dimensional human body and the product development is interrupted, a good fit can be achieved just with numerous iterations that account for time- and labourconsuming process.

The clothing industries emanate so far from an idealized customer's figure, which could not reflect to all the customer groups. As a result, the available pattern making know-how is not adequate to the requirements of different customer groups. The existing systems base on a limited number of 2D body measurements. The complex body shape is deficiently considered in the pattern cutting system. For a good fit, knowledge about the physical structure of human body is of crucial importance. People with almost identical body dimensions may have quite different body shapes. Consequently, the garment fit of different customers in the same size looks different.

With the aid of 3D body scanners, fit relevant information for the garment design such as threedimensional body shape, body posture, body cross-sections, and curves and surfaces can be generated [1].

On account of this 3D body data are implemented in this research work for developing an innovative method of 3D construction of loose fitting clothing, especially trousers. The trouser construction bases on virtual lower bodies of women.

Prerequisites are the evaluation and classification of female 3D scanned data and the development of scalable 3D virtual models. In doing so the classification of body sizes is no more relevant of breast circumference because the virtual models are considered just from the waist down. According to the morphological analysis, hip circumference is suitable to specify as the basis for the anthropometrical characterization of the lower bodies [1]. 


\section{Computer-aided 3D garment simulation and construction}

\subsection{Virtual mannequin}

Virtual CAD-models, which can be applied in 3D CAD systems as design tools, are fundamental necessity for the three-dimensional prototyping [2]. Since the human body structure is quite complex, mathematical consideration and graphical representation play a vital role to attain the realistic description.

The objective of our research is to generate appropriate 3D virtual models of lower bodies from scan data of different body types as a precondition for computer-aided 3D product development (e.g. trouser).

Despite the increased application of body scanners for capturing body dimensions and shapes as 3D data, the clothing industry still prefers to use 2D-CAD tools.

\subsection{Three dimensional garment simulation}

By using 3D fit simulation it is possible to reduce garment sample making and minimize the associated personnel and material costs. For the simulation process two-dimensional pattern pieces must be correctly sewn in a virtual environment relating the corresponding fabric or draping properties. By this way the technological realization of the desired model can be judged virtually. If necessary, modification or changes can be done in two dimensional systems. Finally the garment is again virtually animated and the fit simulation is also reassessed [3].

\subsection{Three dimensional garment construction}

With the aid of 3D CAD software, the desired design can be directly constructed on a virtual model and the corresponding cutting patterns are automatically flattened into the plane. An example of 3D CAD software is Design Concept 3D software (DC3D) [4], which was initially developed for the automotive industry, the upholstered furniture industry and the composites. The application of this software in constructing close-fitting garments has been successfully tested (see figure 1). For closefitting garments, the pattern lines and curves can be carried out on the surface of the virtual model by using drawing tools. The corresponding 2D patterns are then derived automatically [2].
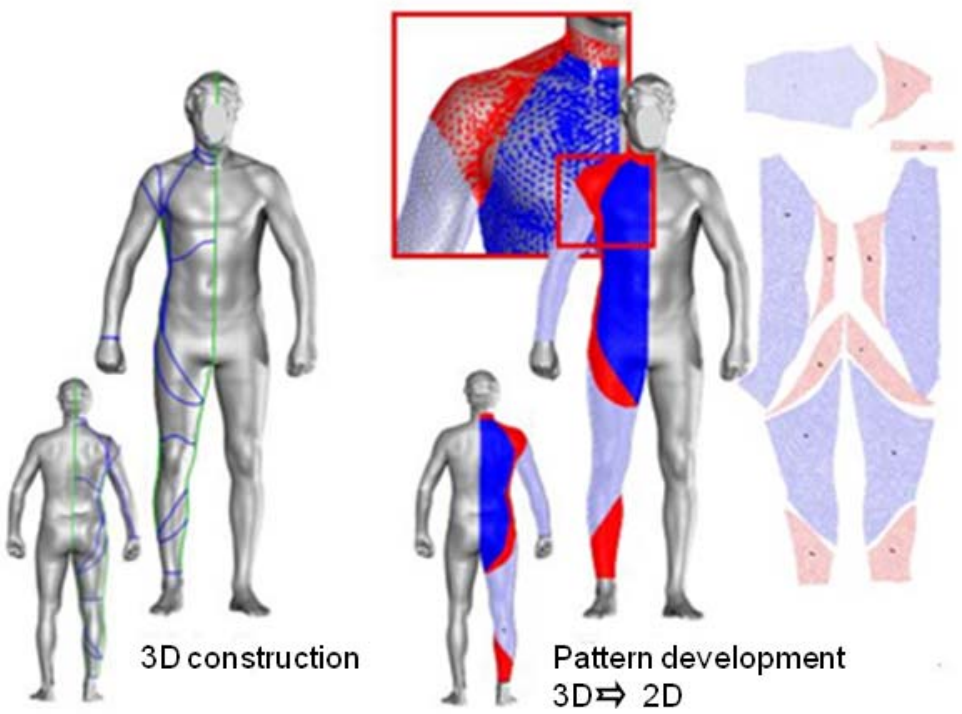

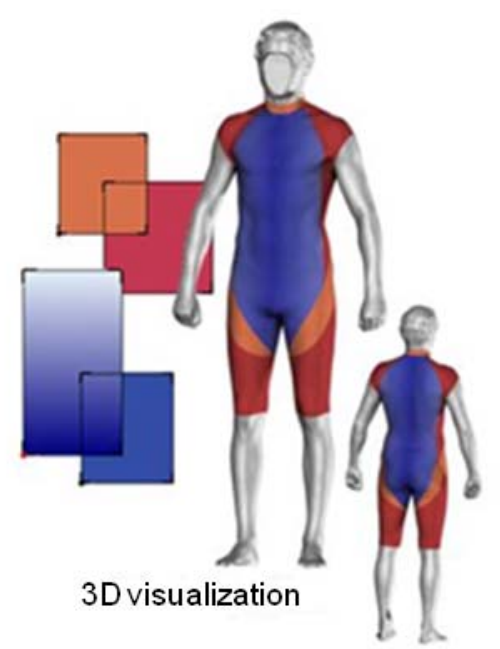

Fig. 1. Development of close-fitting sport clothing [5].

Up to now the available 3D construction method is only suitable for close-fitting garments. To accomplish the construction procedure for loose-fitting trousers efficiently, it is necessary to develop 3D construction algorithm. Hence the objective is to generate a virtual "second skin" with the distances between body and trouser in order to ensure the desired comfort and fulfil fashionable/functional demands. According to morphological changes the whole process proceeds automatically up to $2 \mathrm{D}$ patterns and thus corresponds to a grading in 3D. 


\section{Approach}

\subsection{Classification of the scan data}

The primary requirement for the generation of virtual human models from scan data is the size related classification of these data. The determination of the basic dimensions or body sizes is based on the hip circumference. As the individual human body shapes within the same size differ from each other, it is essential to cluster the body sizes and body types. According to recommendation of the Hohenstein Institute for Textile Innovation, Germany (HIT), the body types corresponding to four waist types (narrow, normal, wide, extra wide) within the same hip circumference are distinguished (see figure 2) [1].

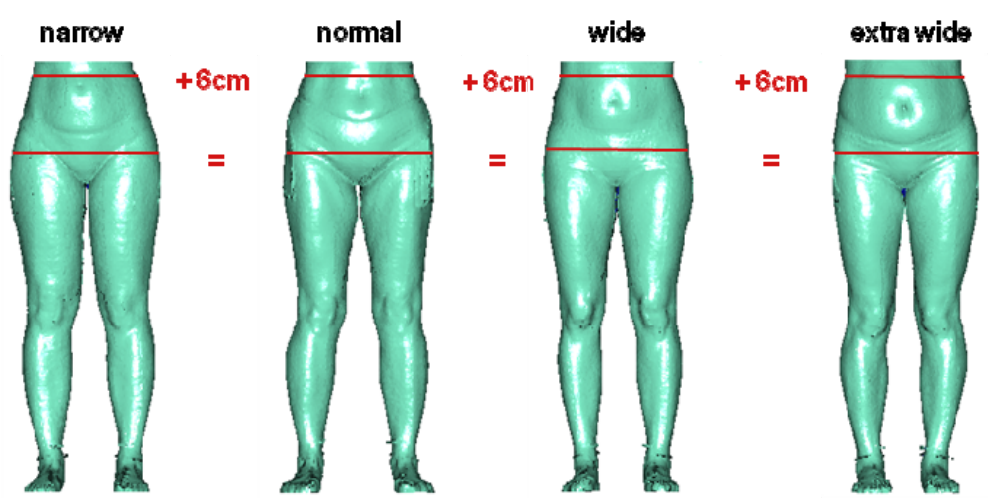

Fig. 2. Defining the waist types at constant hip circumference (e.g. hip circumference - $99 \mathrm{~cm})[1]$.

\subsection{Data selection}

Firstly 3D virtual lower bodies are developed by using personal individualized 3D scan data. For that purpose available sizes are $36,38,40,42,44,46$ with varying waist types (narrow, normal, wide and extra wide) and normal body height. These data are processed with the software Geomagic Studio [6], in order to generate polygon surfaces. Due to the fact that the scan data in shaded areas such as the crotch of the body did not fulfil the required quality, polygon data have to be modified (see figure $3)$.

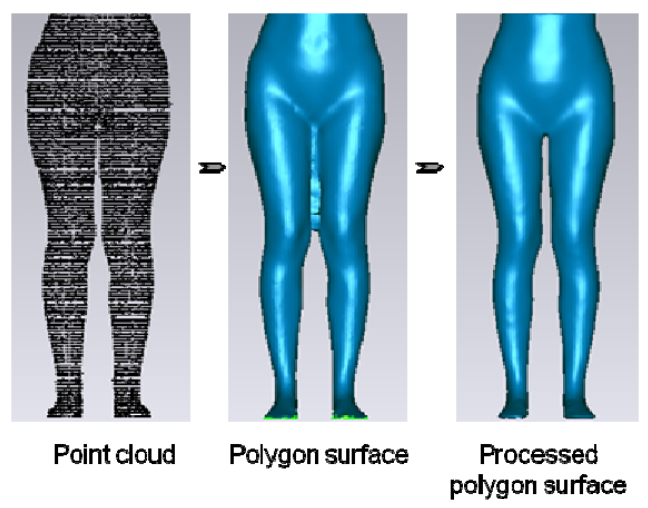

Fig. 3. Transformation of point cloud into polygon surface.

After accomplishing the transforming of point cloud surface into polygon surface, the data are analyzed with regard to the requirements of the virtual or physical model such as body posture and leg position. Afterwards the optimal data are chosen (see figure 4). 


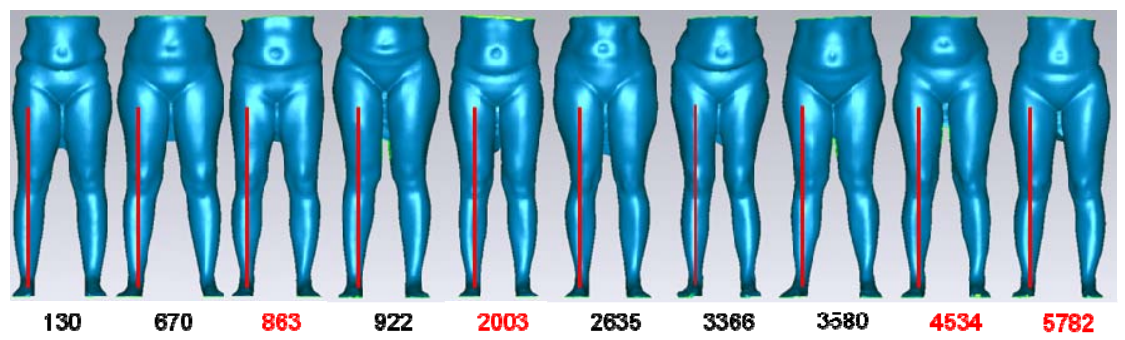

Analysis the front view of body posture (size 45-normal-normal)

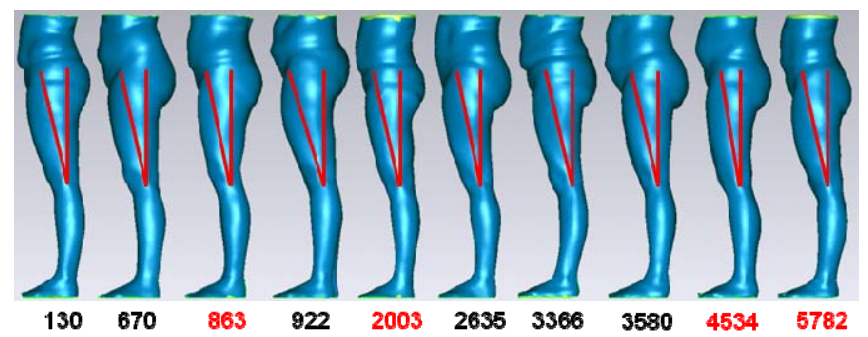

Analysis the side view of body posture (size 46 -normalnormal)

Fig. 4. Data selection (e.g. size.46-normal-normal).

The selected data are then averaged with the aid of software to generate the representative or reference model (see figure 5). Subsequently the body dimensions are verified. If necessary, those have to be scaled according to size chart (HIT) so that the exact dimensions would be attained. Since average virtual model is developed form the unsymmetrical human bodies, the resultant model is asymmetric. For this reason, the optimal side (left or right) is selected and mirrored so as to achieve the symmetrical one. Moreover the hip region is also modified to realize the buttock profile.

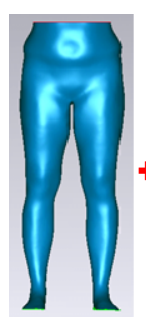

863

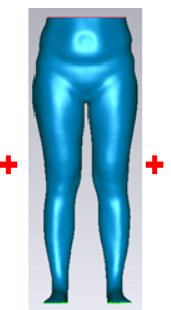

2003

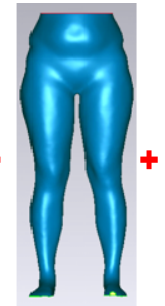

4534

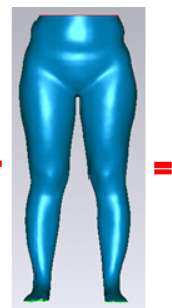

5782

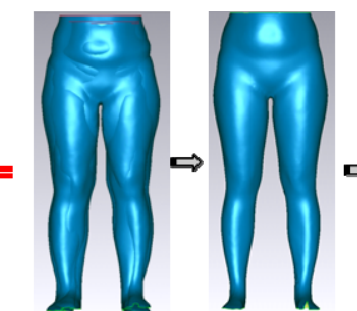

U
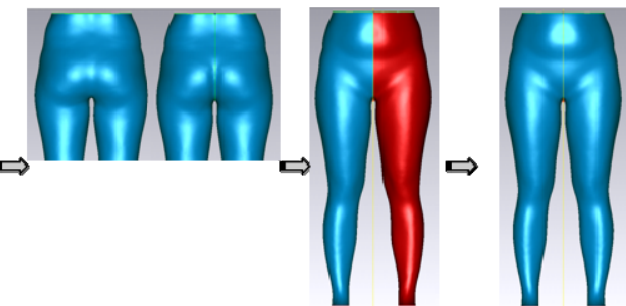

Selected data Averaging Asymmetrical

Modifying the Determining the Representative

Fig. 5. Generation of the representative model by averaging more Data.

For the purpose of 3D construction for loose-fitting garment, it is necessary to convert the polygon surfaces into B-spline surfaces (see figure 6). These can further be implemented as a basis for the trouser construction in commercially available 3D-CAD systems.

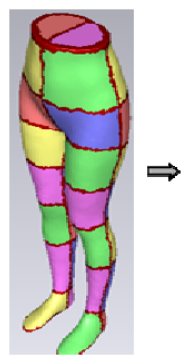

Patches

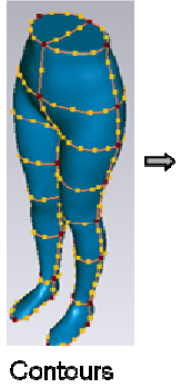

Fig. 6. Developing the NURBS surface.

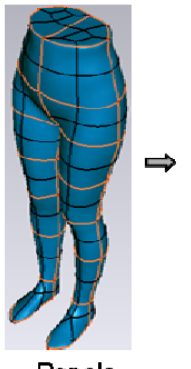

Fine grating

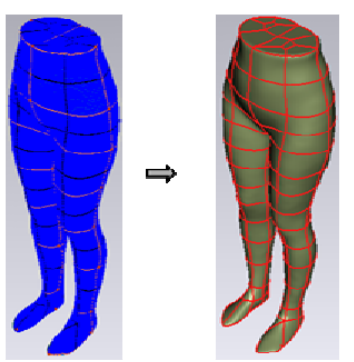

NURBS surface
(1) 


\subsection{Developing the parametric models}

The next step is to generate the parametric $3 \mathrm{D}$ virtual models of various sizes and waist types. For this purpose the reference model which was transformed into B-spline surface is imported to software DC3D [4]. Afterwards the half part of the model is cut with horizontal planes (see figure 7.a) at the required circumferences (primary body dimensions). The cut horizontal slices consist of two types of curves, the opened curve such as waist or hip circumference and the closed curve e.g. thigh circumference. Each sectional curves are measured and created as length parameters so as to scale the circumference of each body dimension in accordance with the size chart (HIT). For the opened curve, the end points are connected and the scaling point is set at one third of this connected line from the back part (see figure 7.b). Contrary to the opened curve, the extreme points of the respective closed sectional curves are automatically found in $x$ and $y$ direction (see figure 7.c) with the aid of the software. These extreme points are then joined together so that the scaling point/centre is determined. For the sake of scaling the virtual model in longitudinal direction, all of the horizontal slices are projected at the crotch point and then duplicated in longitudinal direction $( \pm)$ in a required distance according to the size chart [HIT]. With the intention of the desired profile of the body surface, additional sectional curves are inserted whose parameters are dependent on the primary body dimensions. Thereafter the surface of the virtual model is generated and mirrored to obtain a symmetrical one (see figure 7.d).

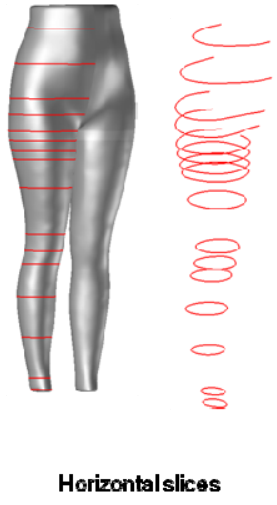

(a:

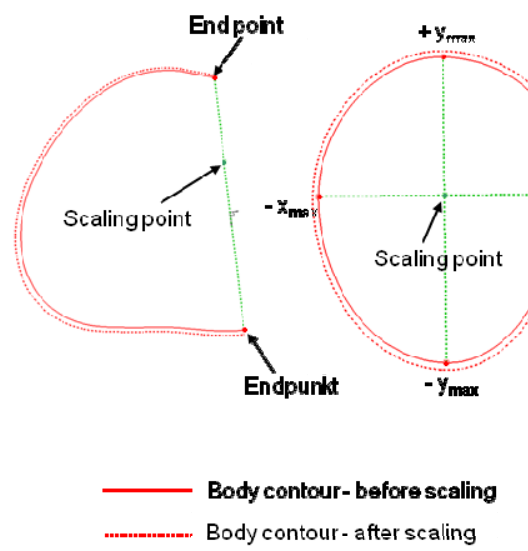

(b) (c)

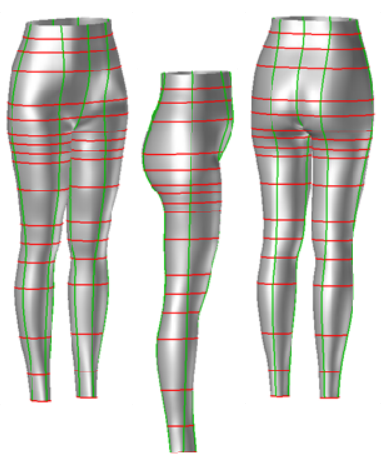

Surface generation andmirroring

Skelaton of the parametric vitualmodel

(d)

Fig. 7. Processing the virtual Model.

Owing to the different morphologies in the hip area, the basic sizes for the scaling of size ranges including four waist types are divided into two groups, namely the size range $38-42$, which is derived from the size 40-normal and size range 44-48 from the size 46-normal. The comparison of the automatically derived virtual models and the reference polygon models are illustrated in figure 8 . If necessary the body posture and leg position are adjusted.

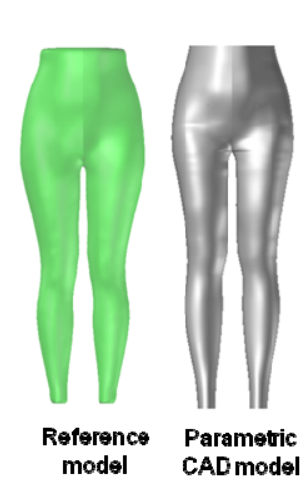

model

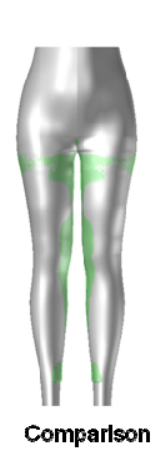

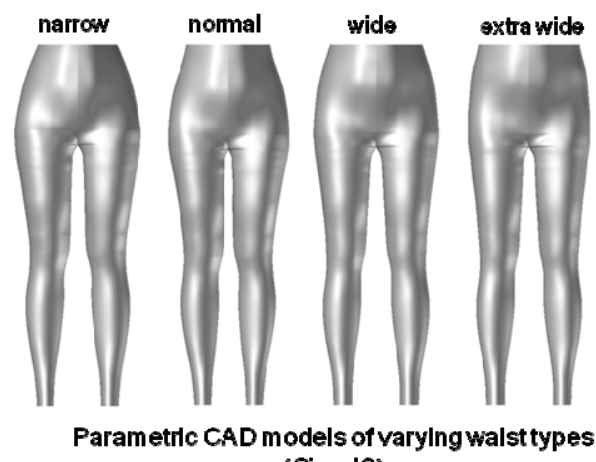

(Size 40)

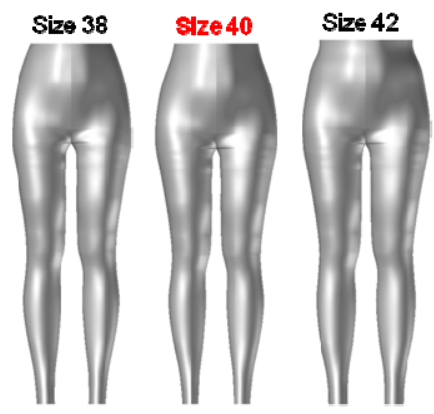

Scallng of virtual models within the same waist type (normal)

Fig. 8. Derivation of scalable parametric virtual models. 


\subsection{Trouser construction}

Before constructing trouser patterns in $3 \mathrm{D}$, it is requisite to simulate two dimensionally constructed trousers on the polygon model, so as to determine the offsets between the virtual model and trouser. The virtually simulated trouser and polygon model are transformed into B-spline surfaces and then imported to the software DC3D (already mentioned for virtual model in section 3.3). Those predefined offsets provide the frame work for constructing the trouser (see figure 9). Subsequently the shell of the simulated trouser is cut at the same level like the virtual model. The construction of the sectional curves and setting the scaling points are analogous to virtual model (see section 3.3).
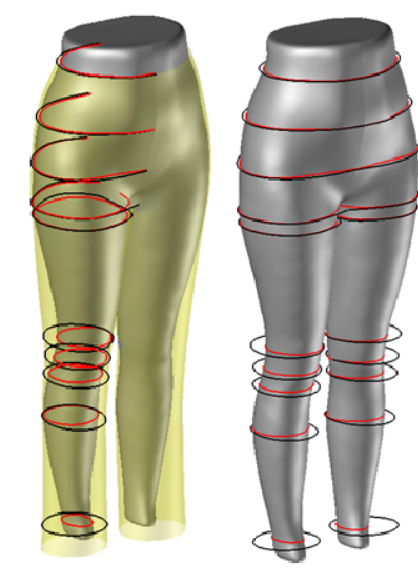

Simulated model and trouser

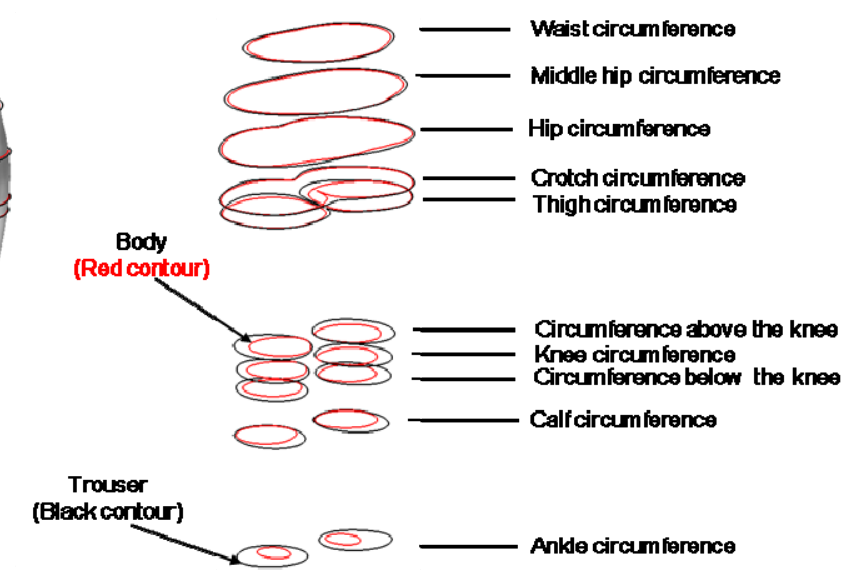

Slices of body and trouser

Fig. 9. Fit simulation for determining the trouser offset

In order to develop the trouser construction, the connected lines of the extreme points of the closed curves (see section 3.3) are extended with the intention that intersection points give rise at the trouser contour (see figure 10.a). The resultant offset between the body and trouser is defined as distance parameter, $V_{1}$ (see figure 10.a). Afterwards the body dimension is scaled according to the standard size chart of HIT [1]. The body contour after scaling is illustrated with red dotted line. In doing so the length of the offset, $V^{\prime}{ }_{1}$ remains constant. Through the connection of the resultant end points of the extended lines, the trouser contour is created. In order to construct the trouser contour exactly, more vectors must be generated (see figure 10.a). For the opened curve the extreme points are automatically developed on the respective sectional slices. Afterwards the end points, the determined extreme points are connected with the scaling point (see figure 10.b). The remaining procedures are accomplished analogous to those of closed curves. By this way the whole trouser contour is exactly generated like the original trouser contour.

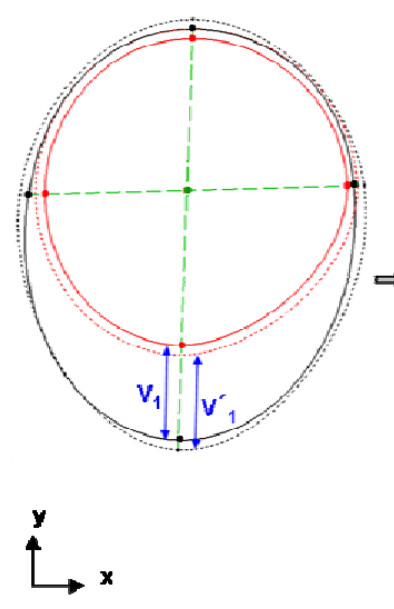

(a) Calf circumference

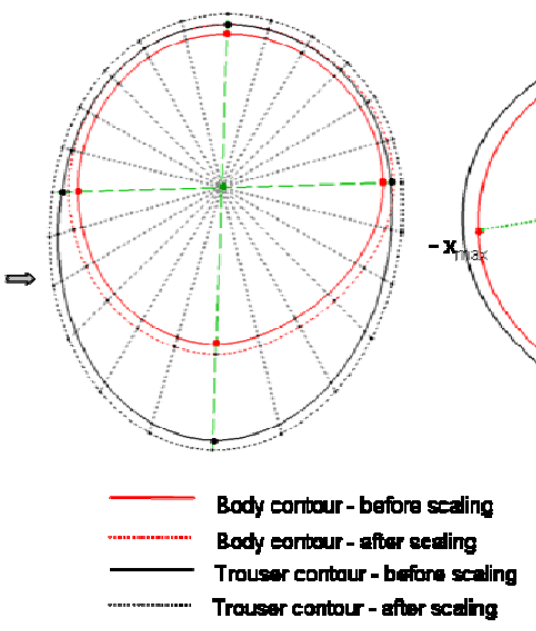

(b) Half of the waist circumference

Fig. 10. Creation of trouser contours. 
For the purpose of developing the trouser surface, the side seam lines as well as crease lines for the front and back parts are constructed. The offsets can be varied by coupling with parameters up to a desired trouser shape (see figure 11). The generated virtual model and garment design that corresponds to a basic design in $3 \mathrm{D}$ can be offered to the garment industry. The task of the designer or stylist is only to define the intended pattern design on the created trouser shell. Therefore this methodology is very suitable also for pattern design makers who are not so skilled in computer technologies.
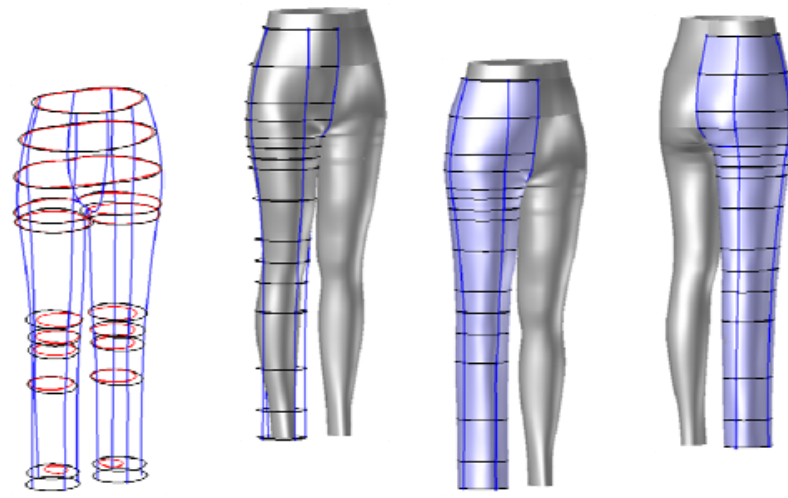

Fig. 11. Trouser construction.

\subsection{Defining the pattern design}

With the intention of flattening the 3D garment patterns into two dimensional planes the constructed 3D patterns are triangulated (see figure 12). The triangulation or meshing provides the approximation of the constructed trouser surface. As the trouser surface is not a ruled surface, the flattening process accomplishes depending on the curvature of the body with minor extensions or reductions of the triangles of the mesh. The boundaries of the trouser are successively activated so that the specific area such as the front or the back part is defined. Subsequently the darts for the front and back trouser parts are also constructed.

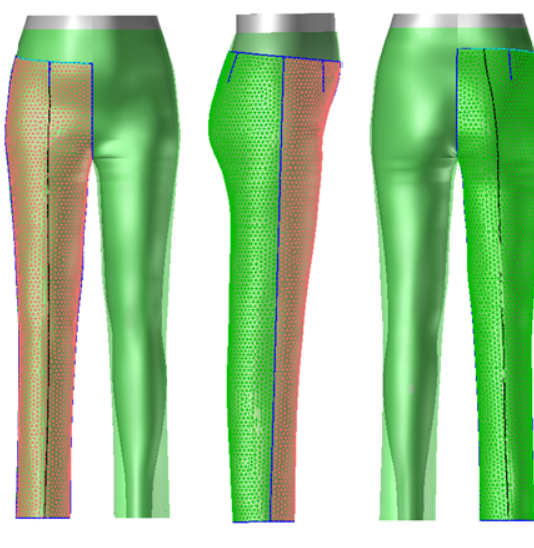

Generating the triangulated mesh

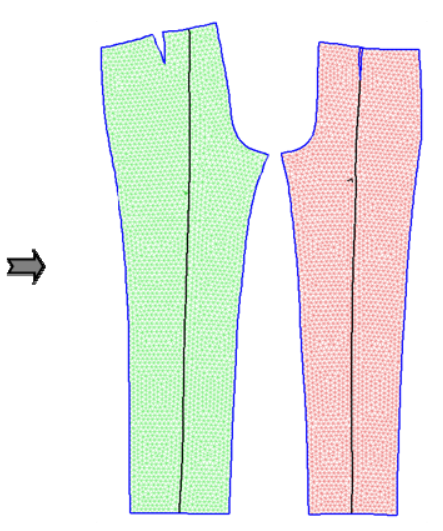

Flattening into 2D patterns

Fig. 12. Automatic generation of 3D-surface into 2D-cutting patterns, size 40-wide-normal.

\subsection{Morphological grading in 3D}

After accomplishing the 3D pattern design and meshing, the virtual mannequins in the basic sizes 40 and 46 are scaled to the nearest sizes $(38,42$ and 44,48$)$ with four various waist types with the aid of Excel sheets, in which the parameters of respective sizes and waist types are stored. Finally the respective 2D garment patterns according to the morphological changes automatically developed without the user intervention.

The overview of the scaled trousers in size spectrum with four waist types as well as automatically generated grading nest with normal waist type are demonstrated in figure 13 (a) and (b) respectively. This can be seen that the dart positions of the two scaling groups are slightly offset owing to the different morphologies of the human bodies. The fit tests for standard and individual sizes have been carried out based on prototypes and the results are good evaluated. 


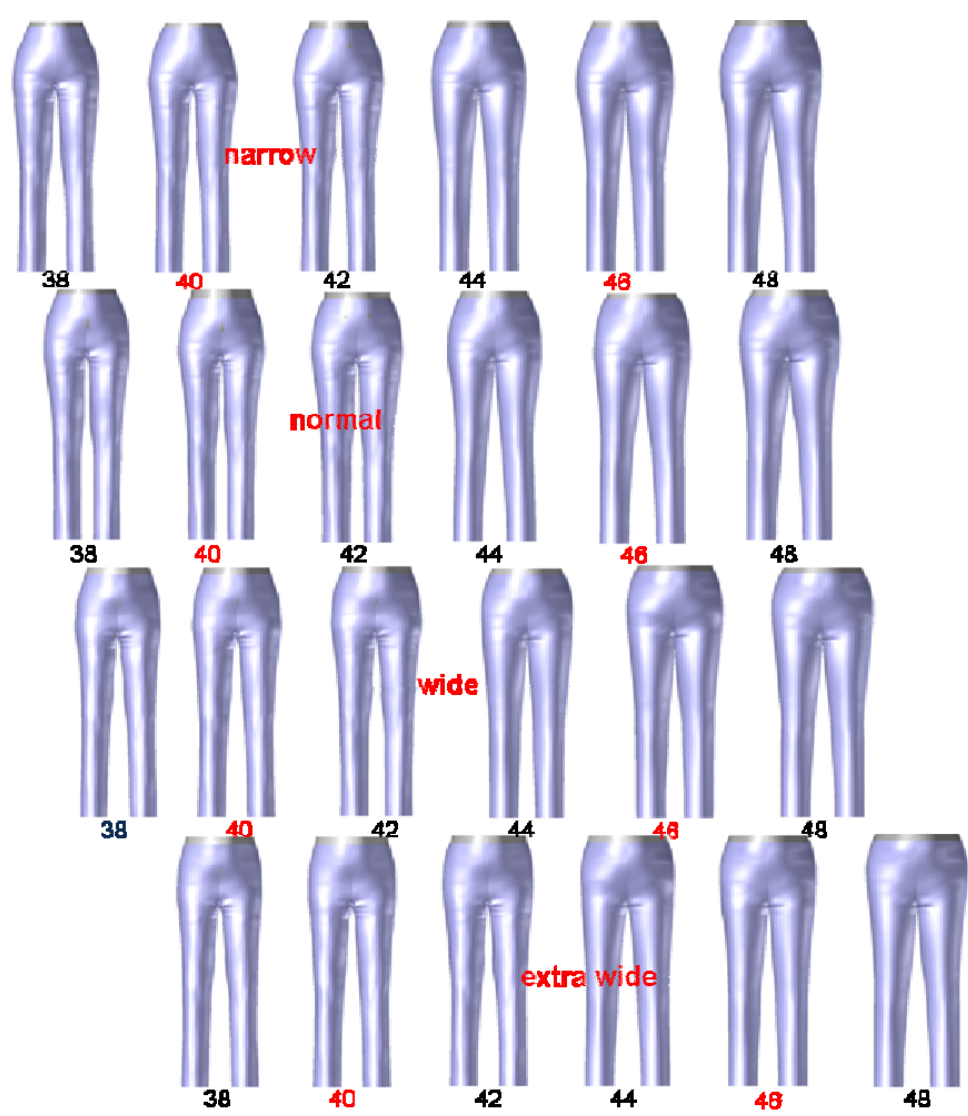

(a)

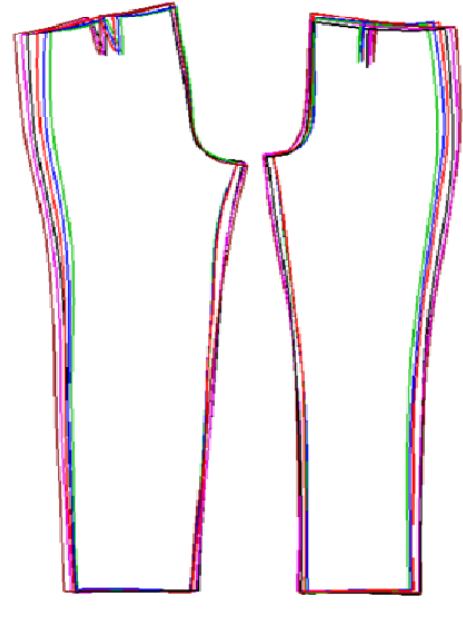

(b)

Fig. 13. (a) Grading of trousers in 3D, (b) 2D trouser patterns of size range (38-48), waist type-normal.

\subsection{Trouser modelling}

In order to create different varieties of fashionable/functional trousers, the trouser shell can be modified by changing the offset parameters by coupling with the Excel sheets and adapted to the desired dimensions. Examples of two constructed models are shown in figure 14 and 15.
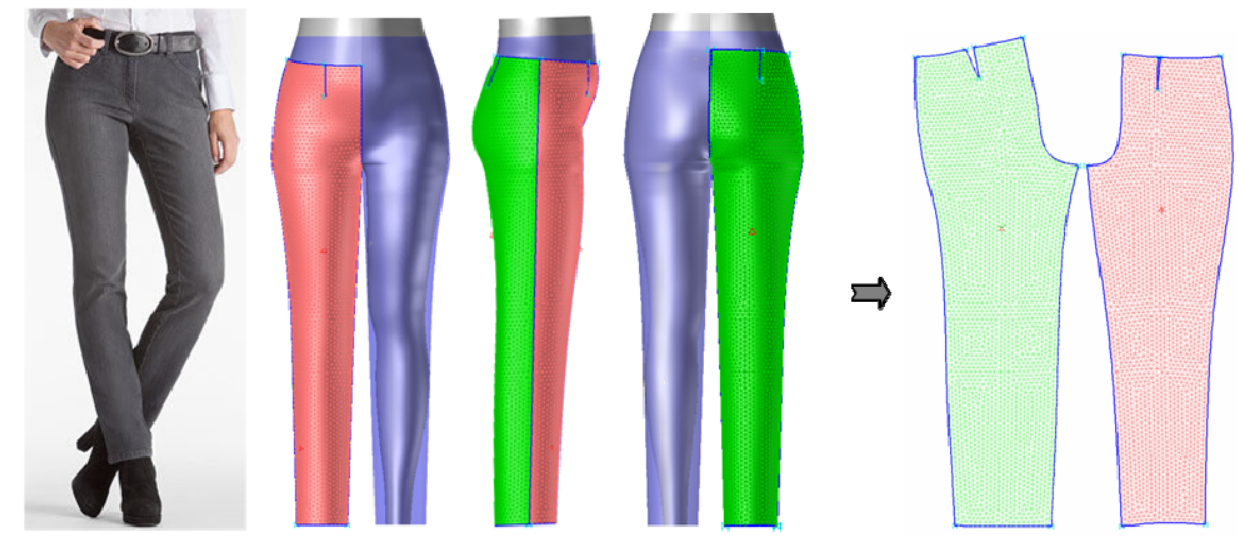

Source: shop.brax [7]

Fig. 14. Model 1, size 40-normal-normal. 


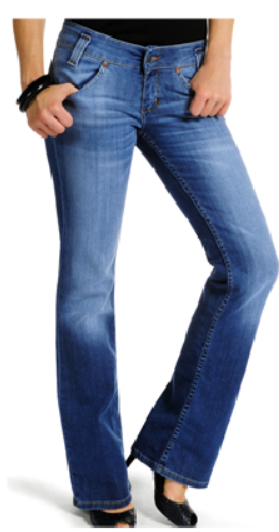

Source: lee-store [8]
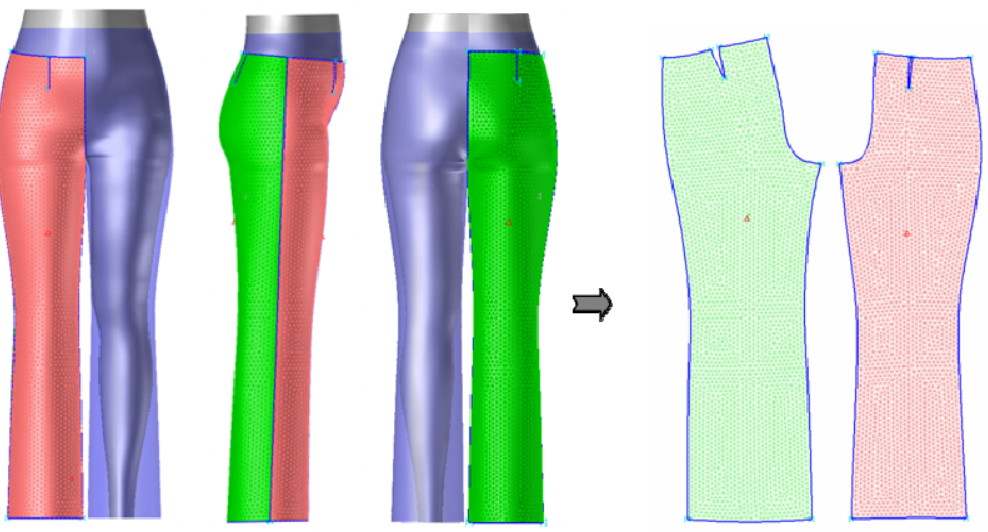

Fig. 15. Model 2, size 40-normal-normal.

\section{Conclusion}

Up to now the garment pattern making can be accomplished through the empirical experience of the designer because there is a lack of the correlation between virtual model and garment pattern. This deficiency can be overcome through the direct construction on the virtual mannequin so as to rationalize the product development significantly.

The desired research's outcomes will provide the indispensable basis of an effective, new technology for the fit relevant construction of loose-fitting garments and thus to further acceleration of the textile chain. Based on the results of the research project it enables optimal cloth fitting corresponding to the different body types for the targeted groups. This leads to an increase of customer satisfaction, a higher purchase frequency and thus contributes significantly to ensure the market success in this segment. As product development remains one of the most cost-intensive processes, the targeted mission of modern technology will bring about savings of up to $60 \%$ of the currently necessary labour time.

\section{References}

1. Krzywinski, S., Hlaing, E.C., Morlock, S., Kirchdörfer, E., (2011): "Entwicklung virtueller 3DFormkörper für die untere Körperhälfte von Frauen auf Basis von 3D-Scandaten", Forschungsbericht, IGF-Forschungsvorhaben Nr. 15972 BG, Institut für Textilmaschinen und Textile Hochleistungswerkstofftechnik, Technische Universität Dresden.

2. Rödel, H., Krzywinski, S., Siegmund, J., (2004): "Schnittgestaltung für körpernahe Bekleidung aus Maschenwaren mit Elastangarnen", Abschlussbericht AiF- Nr. 13190 BR, Institut für Textilmaschinen und Textile Hochleistungswerkstofftechnik, Technische Universität Dresden.

3. Krzywinski, S., (2005): "Verbindung von Design und Konstruktion in der textilen Konfektion unter Anwendung von CAE", Habilitationsschrift, TU Dresden, Fakultät Maschinenwesen.

4. Lectra (accessed 2011): http://www.lectra.com.

5. Krzywinski, S., Rödel, H., Hlaing, E.C., Leipner, A., Siegmund, J., Wendt, E., (2010): "Innovative Produktentwicklung für Bekleidung - 3D Design", IMB Select 2010 "Fashion \& Textiles: IT Systems, Services and Strategies", Köln.

6. Geomagic, "Die führende Lösung zur Flächerrückführung und Inspektion”, (accessed 2010): http://www.geomagic.com/de.

7. BRAX/Leineweber GmbH \& Co. KG (accessed 2011): www.shop.brax.de.

8. Dress-for-less $\mathrm{GmbH}$ (accessed 2011): www.lee-store.de. 